\title{
Oxidation of KCNB1 potassium channels triggers apoptotic integrin signaling in the brain
}

\author{
Wei Yu${ }^{1}$, Manasa Gowda ${ }^{1}$, Yashsavi Sharad ${ }^{1}$, Surindo A Singh ${ }^{1}$ and Federico Sesti ${ }^{*, 1}$
}

Oxidative modification of the voltage-gated potassium $\left(\mathrm{K}^{+}\right)$channel KCNB1 promotes apoptosis in the neurons of cortex and hippocampus through a signaling pathway mediated by Src tyrosine kinases. How oxidation of the channel is transduced into Src recruitment and activation, however, was not known. Here we show that the apoptotic signal originates from integrins, which form macromolecular complexes with KCNB1 channels. The initial stimulus is transduced to Fyn and possibly other Src family members by focal adhesion kinase (FAK). Thus KCNB1 and integrin alpha chain V (integrin- $\alpha_{5}$ ) coimmunoprecipitated in the mouse brain and these interactions were retained upon channel's oxidation. Pharmacological inhibition of integrin signaling or FAK suppressed apoptosis induced by oxidation of KCNB1, as well as FAK and Src/Fyn activation. Most importantly, the activation of the integrinFAK-Src/Fyn cascade was negligible in the presence of non-oxidizable C73A KCNB1 mutant channels, even though they normally interacted with integrin- $\alpha_{5}$. This leads us to conclude that the transition between the non-oxidized and oxidized state of KCNB1 activates integrin signaling. KCNB1 oxidation may favor integrin clustering, thereby facilitating the recruitment and activation of FAK and Src/Fyn kinases.

Cell Death and Disease (2017) 8, e2737; doi:10.1038/cddis.2017.160; published online 6 April 2017

The delayed rectifier, voltage-gated potassium $\left(\mathrm{K}^{+}\right)$channel KCNB1, carries a major somatodendritic current in the cortex and hippocampus. ${ }^{1,2}$ Loss-of-function mutations in KCNB1 have been linked to early infantile epileptic encephalopathy ${ }^{3-5}$ and KCNB1 knock out in mice causes hippocampal hyperexcitability and seizures. ${ }^{6}$ Additionally, during conditions of oxidative stress, reactive oxygen species (ROS) act to modify KCNB1 channels in a manner that they become cytotoxic. ${ }^{7-10}$ For example, in mouse model of traumatic brain injury (TBI), oxidized KCNB1 channels contribute to tissue damage and consequent behavioral impairment. ${ }^{9}$ Oxidized KCNB1 channels are also present in the brains of aging mice and in larger amounts in the brain of 3x-Tg-AD mouse model of Alzheimer's disease, ${ }^{8,11-16}$ where they promote hyperexcitability and presumably apoptosis. ${ }^{17}$ Thus oxidative modification of the KCNB1 channel represents an important mechanism of neuronal vulnerability that has the potential to affect multiple processes in the brain and in the other organs where KCNB1 operates, including the pancreas. ${ }^{18}$ At the molecular level, oxidants crosslink KCNB1 subunits to each other through disulfide bridges involving cys73 in the N-terminus. ${ }^{8}$ These KCNB1 oligomers do not conduct current and are poorly endocytosed. ${ }^{7}$ The accumulation of KCNB1 oligomers in the plasma membrane that follows is associated with the activation of Src tyrosine kinases and downstream C-Jun $\mathrm{N}$-terminal kinases (JNKs), which act to destabilize mitochondria leading to further ROS leakage and finally apoptosis. ${ }^{7,9}$ The molecular steps that link oxidized KCNB1 channels to downstream recruitment and activation of Src tyrosine kinases are not known and this question is significant, considering the role that oxidation of this channel seems to have for normal aging and disease. Previous studies have established a mechanistic link between KCNB1 and focal adhesion kinase (FAK) in the processes associated with cell motility. ${ }^{19,20}$ Thus Wei et al. ${ }^{19}$ showed that KCNB1 can induce FAK autophosphorylation at tyr397 and in this manner control cell polarization and migration. It is well established that autophosphorylation of FAK at tyr397 promotes its association and activation of Src kinases, ${ }^{21}$ which also have a key role in the apoptotic pathway activated by oxidation of KCNB1. This evidence, along with the fact that the FAK-activating signal originates in the first 50 amino acids of the $\mathrm{N}$-terminus of $\mathrm{KCNB} 1,{ }^{19}$ a region not distant from cys 73 , led us to speculate that FAK signaling might have a role in the apoptotic mechanisms associated with oxidation of the channel. Here we show that KCNB1 and integrins form stable complexes in the membranes of neurons of the mouse brain and of mammalian cells. The changes that occur when the channel transitions from a non-oxidized to an oxidized state stimulate integrin signaling leading to autophosphorylation of FAK at tyr397 and consequent activation of Fyn, a member of the SrcA sub-family.

\section{Results}

Integrins form stable complexes with KCNB1 channels in $\mathrm{CHO}$ cells and brain. To determine whether integrins and KCNB1 channels can interact and whether these associations are retained following oxidation of the channel, we carried out coimmunoprecipitation experiments in Chinese hamster ovary $(\mathrm{CHO})$ cells transiently expressing human KCNB1 epitope tagged to the HA tag in the C-terminus (we

\footnotetext{
${ }^{1}$ Department of Neuroscience and Cell Biology, Rutgers University, Robert Wood Johnson Medical School, 683 Hoes Lane West, Piscataway, NJ, USA ${ }^{*}$ Corresponding author: F Sesti, Department of Neuroscience and Cell Biology, Rutgers University, Robert Wood Johnson Medical School, 683 Hoes Lane W, Piscataway, NJ 08854, USA. Tel: 732 2354032; Fax: 732 2355038; E-mail: federico.sesti@ rutgers.edu

Received 06.2.17; revised 10.3.17; accepted 13.3.17; Edited by A Verkhratsky
} 

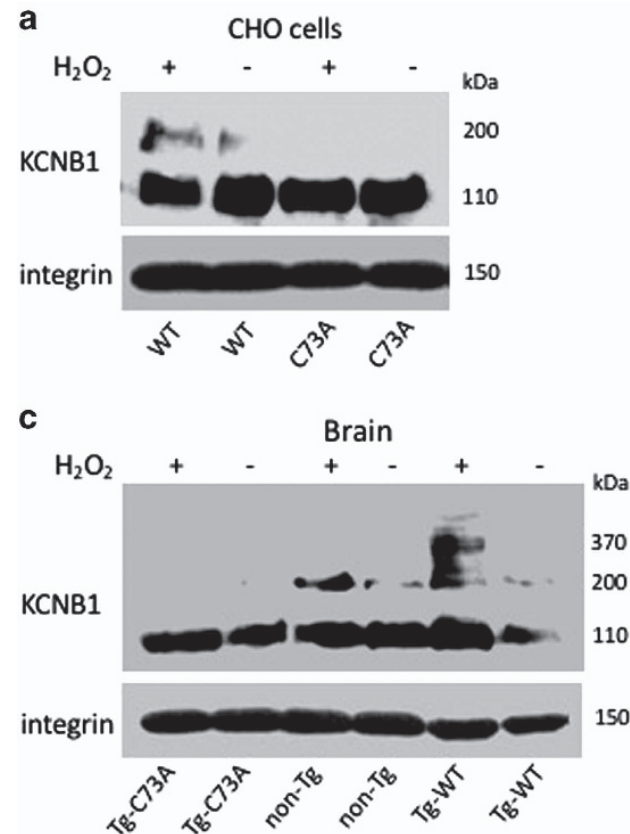

b
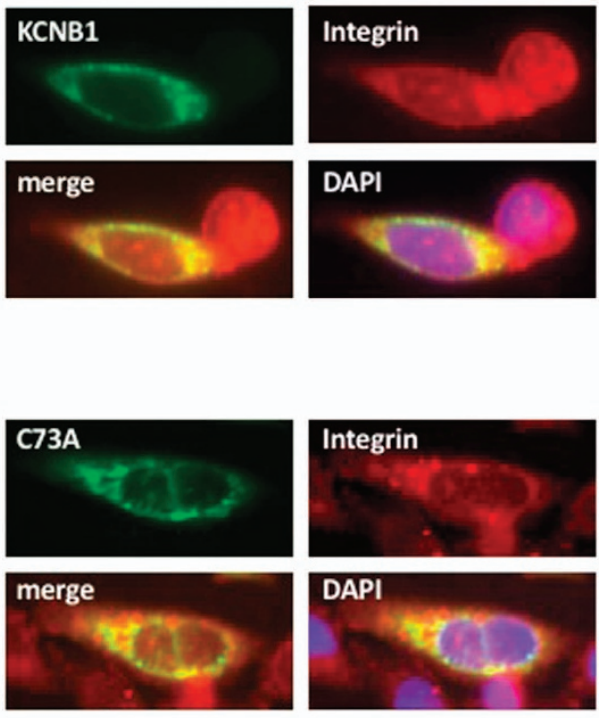

Figure 1 Integrins coimmunoprecipitate with KCNB1 channels. (a) $\mathrm{CHO}$ cells were transfected with cDNA encoding human WT or C73A HA-tagged KCNB1 subunits and lysed in control conditions or subjected to an oxidative insult ( $1.0 \mathrm{mM} \mathrm{H}_{2} \mathrm{O}_{2}$ for $\left.5 \mathrm{~min}\right)$, prior to lysis. In the upper immunoblot, integrin- $\alpha_{5}$ immunoprecipitates were visualized with anti-HA antibodies to detect KCNB1 protein. Total lysates (lower blot) were stained with anti-integrin- $\alpha_{5}$. Integrins were detected in a single $\sim 150 \mathrm{kDa}$ band. (b) Representative images of $\mathrm{CHO}$ cells demonstrating colocalization of WT and C73A with integrins. The cells were transfected with pEGFP-N1-WT or pEGFP-N1-C73A and stained with integrin primary antibody and rhodamine-conjugated secondary antibody. Images were analyzed using the ImageJ software. (c) Brain lysates from the indicated genotypes were treated in control conditions or in the presence of $1.0 \mathrm{mM} \mathrm{H}_{2} \mathrm{O}_{2}$ for $5 \mathrm{~min}$, prior to the addition of sample buffer. In the representative immunoblots, integrin- $\alpha_{5}$ immunoprecipitates were visualized with an antibody against mouse and human KCNB1. Total lysates were stained with anti-integrin- $\alpha_{5}$

showed previously that the addition of the tag has no effect on the properties of the channel ${ }^{8}$ ). KCNB1 was oxidized by exposing the cells to $1.0 \mathrm{mM}$ hydrogen peroxide, $\left(\mathrm{H}_{2} \mathrm{O}_{2}\right)$ for $5 \mathrm{~min}$ prior to lysis. Proteins were immunoprecipitated (IP) with an antibody that detects integrins alpha chain V (integrin-a5) and immunoblotted with a $\mathrm{HA}$ antibody to detect KCNB1 protein. As a control, we used a KCNB1 mutant, $\mathrm{C} 73 \mathrm{~A}$, which does not oligomerize. ${ }^{8}$ Representative western blots of six coimmunoprecipitation experiments are shown in Figure 1. The lower blot in Figure 1a shows staining of total lysates with integrin-a5 antibody. Coimmunoprecipitations are shown in the upper blot in the figure. Oxidized KCNB1 channels in $\mathrm{CHO}$ cells or in the mouse brain form oligomers that can be detected in multiple bands ranging from $\sim 170$ to $\sim 400 \mathrm{kDa}^{8}$ Indeed, integrin-a5 pulled down both the nonoxidized $(\sim 110 \mathrm{kDa})$ and oxidized $(\sim 200 \mathrm{kDa})$ forms of wildtype (WT) KCNB1 indicating that the channel interacted with endogenous integrins in $\mathrm{CHO}$ cells and that these interactions were retained upon its oxidation/oligomerization. Also C73A channels formed a complex with the integrins but in this case only the non-oxidized band was detected in the blot because C73A does not oligomerize.

KCNB1 and integrins colocalize in CHO cells. To further strengthen the notion that KCNB1 channels and integrins form a complex, we assessed their surface colocalization in $\mathrm{CHO}$ cells. For these studies, we employed a green fluorescent protein (GFP)-KCNB1 fusion in the $\mathrm{N}$-terminus (pEGFP-N1-WT or pEGFP-N1-C73A), stained $\mathrm{CHO}$ cells with integrin-a5 antibody and imaged immunofluorescence using Nomarski microscopy. In agreement with coimmunoprecipitation results, strong colocalization of KCNB1 and integrins was detected on the surface of $\mathrm{CHO}$ cells transfected with either pEGFP-N1-WT or pEGFP-N1-C73A (Figure 1b). The lack of strong colocalization in the cells' cytoplasm may indicate that KCNB1 and integrins traffic to the plasma membrane following different routes.

KCNB1 and integrins form stable complexes in the mouse brain. We next determined whether KCNB1 formed a complex with integrins in the mouse brain. Integrin-a5 were IP from the lysates of half-brains of 3-month-old mice treated in the absence/presence of $1.0 \mathrm{mM} \mathrm{H}_{2} \mathrm{O}_{2}$ for $5 \mathrm{~min}$ before the addition of sample buffer and immunoblotted with a KCNB1 antibody that detects a C-terminal epitope conserved in the mouse and human channel. To ascertain whether integrins interacted with C73A mutant channels in the brain, we took advantage of transgenic mice expressing human C73A tagged to the HA tag in the C-terminus (Tg-C73A) in the cortex and hippocampus and, as an additional control, of transgenic mice expressing WT tagged to the HA tag in the C-terminus (Tg-WT) that we previously characterized. ${ }^{9}$ In those animals, exogenous and endogenous KCNB1 subunits appear to form heteromeric complexes (indeed, mouse and human KCNB1 share $97 \%$ amino-acid sequence identity ${ }^{9}$ ). As a result, the amounts of oxidized KCNB1 channels are small in the Tg-C73A brain, because the cys73 to ala mutation is dominant negative while in the Tg-WT brain due 
to overexpression they are larger than in non-Tg. ${ }^{9}$ Thus, in agreement with results obtained using $\mathrm{CHO}$ cells, integrinsa5 formed stable complexes with KCNB1 channels and these interactions were maintained during channels' oligomerization ( 200- $\sim 370 \mathrm{kDa}$ bands, Figure 1c). Similar interactions were detected in the $\mathrm{Tg}-\mathrm{WT}$ and $\mathrm{Tg}-\mathrm{C} 73 \mathrm{~A}$ brains, as expected.

Taken together, these results lead us to conclude that integrins are general partners of KCNB1 channels.

Inhibition of integrins prevents KCNB1-induced apoptosis. The finding that KCNB1 interacts with integrins led us to speculate that the oligomerization of the channel that follows its oxidation might activate integrin signaling, for example, by stimulating their clustering, which drives the recruitment and activation of Src kinases via FAK. ${ }^{22,23}$ If integrins are responsible for the apoptotic stimulus, pharmacologically inhibiting integrin signaling should prevent apoptosis induced by oxidation of KCNB1, whereas inhibiting integrin signaling in cells expressing C73A should have only minor effect on their viability. To test this hypothesis, cells were subjected to an oxidative insult and then incubated in the presence of integrin inhibitor Cyclo (-RGDfK) or vehicle. Early apoptosis was assessed $6 \mathrm{~h}$ postoxidation by Annexin$\mathrm{V}$ staining, as carried out before. ${ }^{7-9}$ Thus cells expressing
WT were susceptible to apoptosis in agreement with previous studies $^{8}$ (Figures $2 a$ and b). Cells expressing the C73A mutant exhibited a significantly lower rate of apoptotic death than cells transfected with WT; in fact, apoptosis levels in C73A-transfected cells were comparable to those of mocktransfected cells (Figure 2c). Cyclo inhibited apoptosis in $\mathrm{CHO}$ cells expressing the WT channel in a dose-dependent manner, with an $\mathrm{IC}_{50}=38.6 \pm 2.3 \mathrm{nM}$ (Figure 2b). Also primary hippocampal neurons obtained from non-Tg and Tg-WT embryos were susceptible to oxidant-induced apoptosis (Figure 2d. For representative images, see Figure 4). In agreement with previous studies, ${ }^{9} \mathrm{Tg}-\mathrm{C} 73 \mathrm{~A}$ neurons retained moderated apoptosis susceptibility probably due to the residual oxidation of the channel in the brain of this genotype. When the cultures were treated with Cyclo, the inhibitor significantly suppressed apoptosis in all genotypes.

Integrins stimulate FAK autophosphorylation at tyr397. To determine whether oxidation of KCNB1 could induce activation of FAK via integrin signaling, we assessed the fraction of autophosphorylated FAK at tyr397 in $\mathrm{CHO}$ cells transfected with WT or C73A. Representative western blots of these experiments along with quantitative analyses are shown in Figure 3a. Thus autophosphorylated FAK proteins were detected in WT-expressing cells that had been exposed a
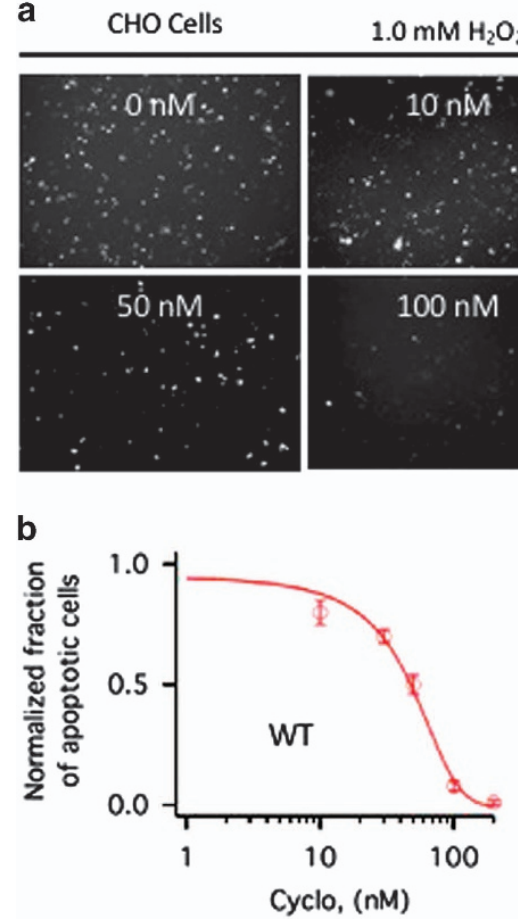

$1.0 \mathrm{mM} \mathrm{H}_{2} \mathrm{O}_{2}$

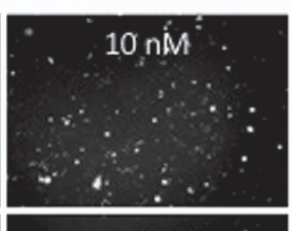

$100 \mathrm{nM}$

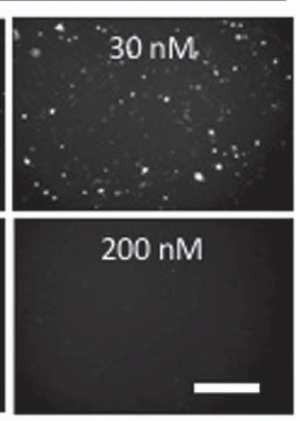

c

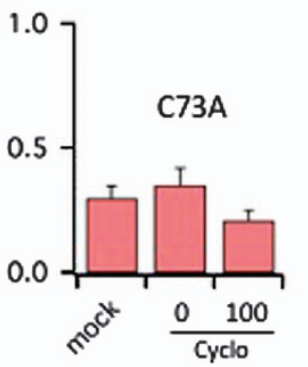

d

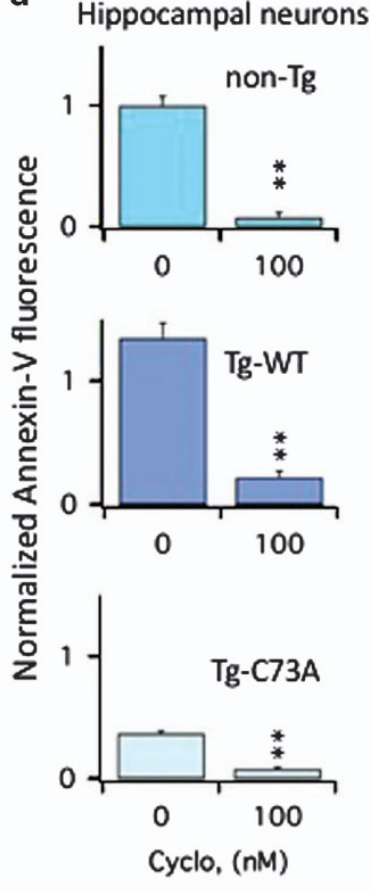

Figure 2 Cyclo protects $\mathrm{CHO}$ cells and primary hippocampal neurons from apoptosis induced by oxidation of KCNB1. (a) Representative examples of Annexin-V staining in $\mathrm{CHO}$ cells transfected with WT. The cells were exposed to $1.0 \mathrm{mM} \mathrm{H}_{2} \mathrm{O}_{2}$ for 5 min and then incubated $6 \mathrm{~h}$ in the presence of Cyclo at the indicated nanomolar concentrations. At the end of the 6-h incubation period, cells were stained with Annexin-V and photographed. Size bar $100 \mu \mathrm{m}$. (b) Number of WT-transfected CHO cells subjected to an oxidative challenge positive to Annexin-V in the absence/presence of varying concentrations of Cyclo. Data are normalized to the number of cells positive to Annexin- $\mathrm{V}$ in the absence of Cyclo. Data were fit to a sigmoidal function (equation (1)) with an $\mathrm{IC}_{50}=41.4 \mathrm{nM}$. $N=3$ experiments. (c) Number of mock or $\mathrm{C} 73 \mathrm{~A}$-transfected $\mathrm{CHO}$ cells positive to Annexin-V subjected to an oxidative challenge in the absence or presence of $100 \mathrm{nM}$ Cyclo. $N=3$ experiments. (d) Quantitative assessment of Annexin-V fluorescence (proportional to the number of cells undergoing apoptosis) in primary hippocampal neurons of the indicated genotypes subjected to an oxidative challenge. After wash out, cultures were incubated in the absence/presence of $100 \mathrm{nM}$ Cyclo for $6 \mathrm{~h}$ and then stained with Annexin-V. Fluorescence was assessed using the Image $\mathrm{J}$ software and data are normalized to non-Tg neurons in the absence of Cyclo. $P=1.8 \times 10^{-10}$ (one-way analysis of variance), ${ }^{* *} P<0.01$ (Tukey's post hoc). $N=3$ embryos/genotype 

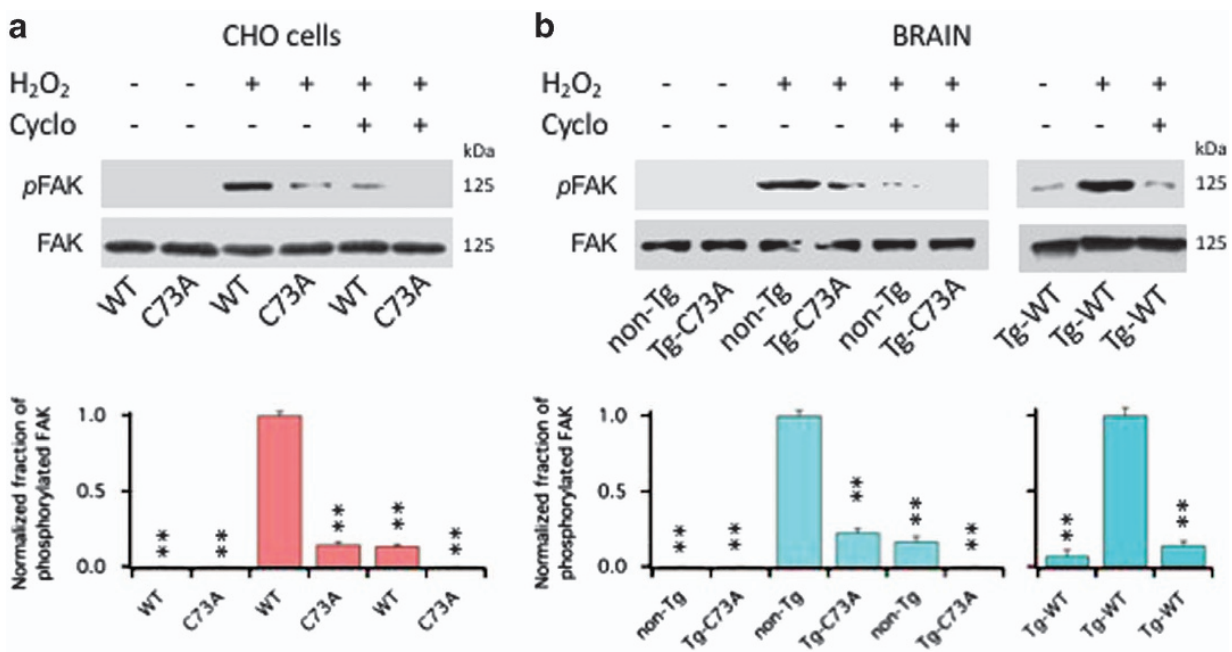

Figure 3 Cyclo inhibits FAK activation induced by oxidation of KCNB1. (a) Representative western blots showing autophosphorylated FAK at tyr397 (pFAK) and total FAK (FAK) in $\mathrm{CHO}$ cells transfected with WT or C73A in the absence/presence of $200 \mathrm{nM}$ Cyclo. FAK protein was detected into a single, $\sim 125 \mathrm{kDa}$ band. Cells were oxidized with $1.0 \mathrm{mM} \mathrm{H}_{2} \mathrm{O}_{2}$ for $5 \mathrm{~min}$ and then incubated in control media or in media containing $200 \mathrm{nM}$ Cyclo for $1 \mathrm{~h}$ before lysis. Quantifications of three experiments are shown in the lower panel and are normalized to $\mathrm{WT}+\mathrm{H}_{2} \mathrm{O}_{2} \cdot P=1.0 \times 10^{-8}$ (one way analysis of variance (ANOVA)). ${ }^{* *} \mathrm{P}<0.01$ for pairwise comparisons versus WT $+\mathrm{H}_{2} \mathrm{O}_{2}$ (Tukey's post hoc test). (b) Representative western blots showing phosphorylated FAK (pFAK) and total FAK (FAK) in the brains of the indicated genotypes in the absence/presence of $200 \mathrm{nM}$ Cyclo. Brain lysates were incubated $1 \mathrm{~h}$ in the absence/presence of $1.0 \mathrm{mM} \mathrm{H}_{2} \mathrm{O}_{2}$ and the absence/presence of $200 \mathrm{nM}$ Cyclo. The reactions were stopped by adding sample buffer to the lysates. For non- $\mathrm{Tg}$ and $\mathrm{Tg}-\mathrm{C} 73 \mathrm{~A}$, quantifications of three experiments are shown in the lower panel and are normalized to non- $\mathrm{Tg}+\mathrm{H}_{2} \mathrm{O}_{2} \cdot \mathrm{P}=1.4 \times 10^{-6}$ (one-way ANOVA). ${ }^{*} \mathrm{P}<0.01$ for pairwise comparisons versus non- $\mathrm{Tg}+\mathrm{H}_{2} \mathrm{O}_{2}$ (Tukey's post hoc test). For Tg-WT, quantifications of two experiments are shown in the lower panel and are normalized to $\mathrm{Tg}-\mathrm{WT}+\mathrm{H}_{2} \mathrm{O}_{2} . P=1.7 \times 10^{-4}$ (one-way ANOVA). ${ }^{* \star} \mathrm{P}<0.01$ for pairwise comparisons versus $\mathrm{Tg}-\mathrm{WT}+\mathrm{H}_{2} \mathrm{O}_{2}$ (Tukey's post hoc test)

to an oxidative insult and in significantly smaller amounts, in cells expressing C73A (Figure 3a). Most importantly, FAK autophosphorylation was markedly reduced in cells exposed to $200 \mathrm{nM}$ Cyclo. Ex vivo experiments with mouse brains, shown in Figure 3b, recapitulated results obtained with $\mathrm{CHO}$ cells (brain homogenates were preferred over primary neurons because the brain provides a more physiological system). Thus autophosphorylated FAKs were detected in lysates of non-Tg brains and Tg-WT brains exposed to an oxidative insult, whereas their amounts were lower in lysates of Tg-C73A brains. In all cases, $200 \mathrm{nM}$ Cyclo significantly decreased FAK autophosphorylation at tyr397. The amounts of autophosphorylated FAK were $\sim 36 \%$ larger in the Tg-WT brains compared with the non- $\mathrm{Tg}$ brains, consistent with the presence of higher amounts of oxidized KCNB1 channels in the former (in the figure, data are plotted normalized to their internal control).

Inhibition of FAK protects against KCNB1-mediated apoptosis. Annexin- $\mathrm{V}$ staining following an oxidative insult was significantly lowered in $\mathrm{CHO}$ cells expressing WT treated with FAK inhibitor PND-1186 (Figure 4a). The effect of the inhibitor was dose dependent with an $\mathrm{IC}_{50}=2.1 \pm 0.1 \mathrm{nM}$. In contrast, the inhibitor had only a marginal effect in cells transfected with $\mathrm{C} 73 \mathrm{~A}$, which are characterized by low levels of apoptosis. The antiapoptotic effect of PND-1186 was further assessed in primary hippocampal neurons subjected to an oxidative insult (Figures $4 \mathrm{~b}$ and $\mathrm{c}$ ). Representative images of Annexin- $V$ staining in the cultures of hippocampal neurons are shown in Figure 4b. Quantification of those experiments, shown in Figure 4c, confirmed that PND-1186 significantly inhibited apoptosis in all genotypes.
Integrins activate Fyn tyrosine kinases. We next determined whether FAK was responsible for the recruitment and activation of Src kinases that follows oxidation of KCNB1. To asses the fraction of activated Src kinases, we used an antibody that detects phosphorylation status of tyr416, a residue conserved in all members of the Src kinase family, as carried out before. ${ }^{9,24}$ Thus, in $\mathrm{CHO}$ cells transfected with WT or C73A, Src phosphorylation at tyr416 was negligible at baseline (Figure 5a). Following an oxidative insult, Src phosphorylation was significantly increased in cells expressing the WT channel compared with cells expressing the C73A mutant, and most importantly, treatments with $200 \mathrm{nM}$ Cyclo or $10 \mathrm{nM}$ PDN-1186 suppressed it. Similar results were observed in the brains of non-Tg, Tg-WT and Tg-C73A mice, with the amounts of phosphorylated Src $\sim 32 \%$ larger in the Tg-WT brains compared with the non-Tg brains (Figure $5 \mathrm{~b}$. In the figure, data are plotted normalized to their internal control). The family of Src tyrosine kinases is composed of nine members, including Fyn, which belongs to the SercA sub-family and has been implicated in TBI and Alzheimer's disease, two conditions associated with robust KCNB1 oxidation. ${ }^{8,9,17,25,26}$ Therefore, we next sought to determine whether oxidation of KCNB1 resulted in the activation of Fyn using a specific antibody that recognizes phosphorylated tyr530 in Fyn. Figure 6a shows the fraction of phosphorylated Fyn kinases in $\mathrm{CHO}$ cells transfected with WT or C73A and Figure $6 \mathrm{~b}$ in the brain lysates of non- $\mathrm{Tg}, \mathrm{Tg}-\mathrm{WT}$ and $\mathrm{Tg}-\mathrm{C} 73 \mathrm{~A}$ in control conditions or following exposure to $1.0 \mathrm{mM} \mathrm{H}_{2} \mathrm{O}_{2}$. The fraction of phosphorylated Fyn protein at tyr530 following an oxidative challenge was significantly increased in all cells expressing the WT channel compared with control and remained low in cells expressing the C73A mutant. Further, 
a

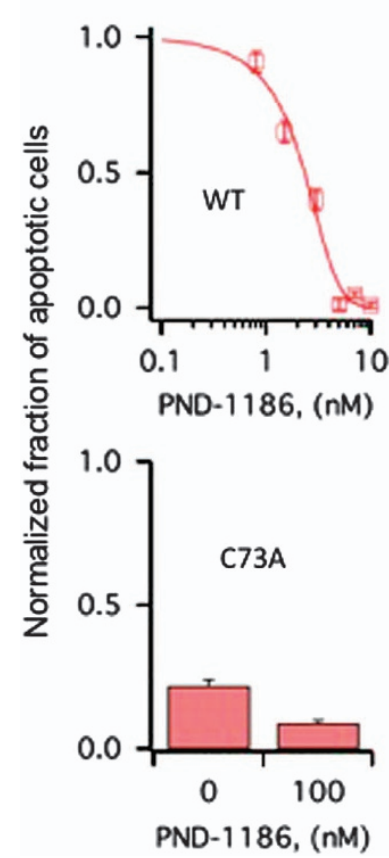

b

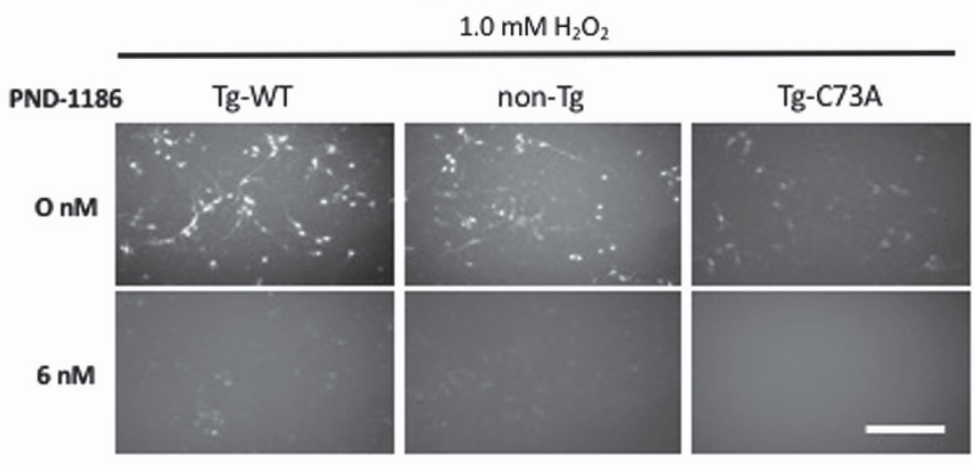

c

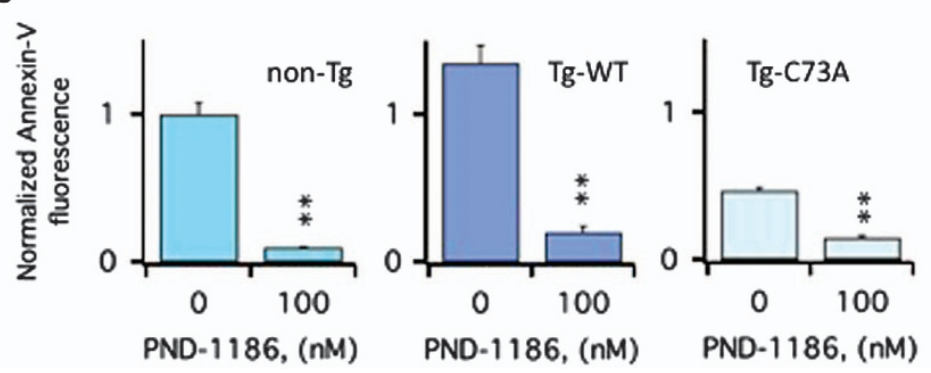

Figure 4 PND-1186 protects $\mathrm{CHO}$ cells and primary hippocampal neurons from apoptosis induced by oxidation of KCNB1. (a) Upper panel: number of cells positive to Annexin-V in WT-transfected CHO cells subjected to an oxidative challenge in the absence/presence of varying concentrations of PND-1186. Data are normalized to the number of cells positive to Annexin-V in the absence of PND-1186. Data were fit to a sigmoidal function (equation (1)) with an $\mathrm{IC}_{50}=1.95 \mathrm{nM}$. $N=3$ experiments. Lower panel: number of C73A-transfected $\mathrm{CHO}$ cells positive to Annexin-V subjected to an oxidative challenge in the absence or presence of $6 \mathrm{nM}$ PND-1186. $N=3$ experiments. (b) Representative examples of Annexin- $V$ staining in the primary hippocampal neurons of the indicated genotypes. The cells were exposed to $1.0 \mathrm{mM} \mathrm{H}_{2} \mathrm{O}_{2}$ for 5 min, incubated $6 \mathrm{~h}$ in the presence of $6 \mathrm{nM}$ PND-1186, stained with Annexin-V and photographed. Size bar $100 \mu \mathrm{m}$. (c) Quantitative assessment of Annexin-V fluorescence in the primary hippocampal neurons of the indicated genotypes subjected to an oxidative challenge in the absence/presence of $6 \mathrm{nM}$ PND-1186. Annexin-V fluorescence was calculated using the ImageJ software and data are normalized to non-Tg neurons in the absence of PND-1186. $P=3.9 \times 10^{-7}$ (one-way analysis of variance); ${ }^{* *} P<0.01$ (Tukey's post hoc). $N=3$ embryos/genotype

Fyn phosphorylation was significantly decreased by treatment with Cyclo or PND-1186.

Together these data led us to conclude that KCNB1 oligomers activate an integrin-FAK-Src/Fyn apoptotic pathway that is conserved in both primary hippocampal neurons and mammalian cells.

\section{Discussion}

In this study, we investigated how oxidation of KCNB1 in neurons of the brain is transduced into an apoptotic signal for Src tyrosine kinases. We found that the apoptotic stimulus originates from integrins, which form macromolecular complexes with KCNB1 channels. Thus KCNB1 and integrin- $a_{5}$ coimmunoprecipitated in the mouse brain and in $\mathrm{CHO}$ cells, and most importantly, these interactions were retained upon channel's oligomerization. Moreover, pharmacological inhibition of integrin signaling in primary neurons and $\mathrm{CHO}$ cells was protective against apoptosis induced by oxidation of KCNB1. Integrins are heterodimers formed by an $\alpha$ and a $\beta$ subunit. We did not attempt to identify which $\beta$-subunit complements integrin- $\alpha_{5}$ in forming a complex with KCNB1, but it is possible that large heterogeneity may exist. For example, integrin- $a_{5}$ can form at least four distinct heterodimers with subunits $\beta_{1}$, $\beta_{5}, \beta_{6}$ and $\beta_{8}$ in cortical neurons. ${ }^{27}$
Integrins were found to signal through FAK kinases, which have been previously shown to work in concert with KCNB1 in pathological mechanisms of cell adhesion and migration. ${ }^{19}$ Accordingly, biochemical evidence indicated that oxidation of WT channels was associated with significant FAK and SrC phosphorylation, which could be suppressed by pharmacological inhibition of integrin signaling. Further, inhibiting the catalytic activity of FAK diminished apoptosis in primary hippocampal neurons and $\mathrm{CHO}$ cells. Integrins activate FAK kinases by stimulating autophosphorylation at $Y 397 .{ }^{23}$ This promotes the formation of a FAK-Src signaling complex through the creation of a high-affinity binding site for the Srchomology 2 ( $\mathrm{SH} 2)$ domain that leads to maximal FAK catalytic activity. ${ }^{28}$ It is not known at this stage whether Src kinases exert their pro-apoptotic action when they are associated with FAK or alone, but in both cases they have broad signaling capabilities. The Src family is composed of nine members and it is therefore possible that multiple kinases are activated by FAK. We found that Fyn, which belongs to the SercA subfamily, has an active role in the pathway under study. The involvement of Fyn is consistent with the prominent role of this kinase in TBI and Alzheimer's disease, two conditions associated with robust KCNB1 oxidation. ${ }^{8,9,17,25,26}$ The amounts of phosphorylated Fyn and Src were comparable, but the proteins were detected by different antibodies and 
a

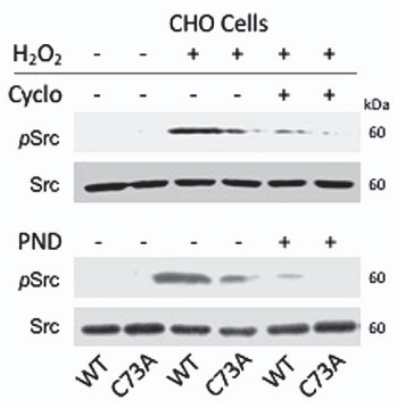

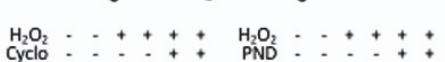

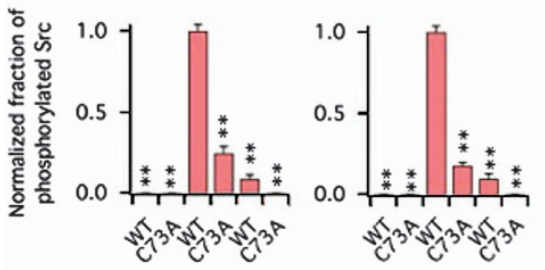

b
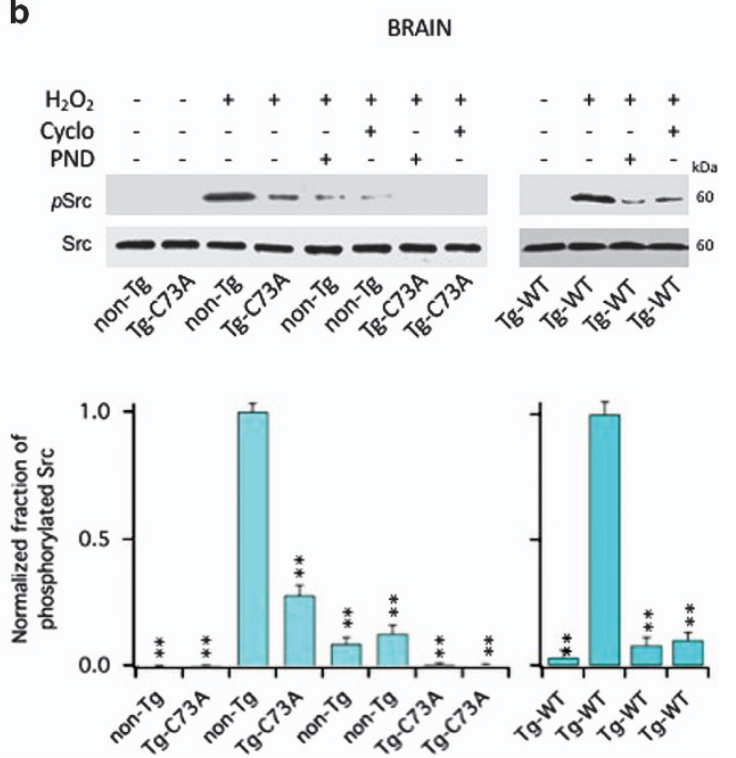

Figure 5 Cyclo and PND-1186 inhibit Src activation induced by oxidation of KCNB1. (a) Representative western blots showing phosphorylated Src at Tyr 416 (pSrc) and total $\mathrm{Src}(\mathrm{Src})$ in $\mathrm{CHO}$ cells transfected with WTor C73A in the absence/presence of the indicated inhibitors. Src protein was detected into a single, $\sim 60 \mathrm{kDa}$ band. Cells were oxidized with $1.0 \mathrm{mM} \mathrm{H}_{2} \mathrm{O}_{2}$ for 5 min and then incubated in control media or in media containing $200 \mathrm{nM}$ Cyclo or $10 \mathrm{nM}$ PND-1186 (PND) for $1 \mathrm{~h}$ before lysis. Quantifications of three experiments are shown in the lower panel and are normalized to $\mathrm{WT}+\mathrm{H}_{2} \mathrm{O}_{2} . P=2.9 \times 10^{-6}$ and $3.5 \times 10^{-6}$ for Cyclo and PND-1186, respectively (one-way analysis of variance (ANOVA)). ${ }^{*} \mathrm{P}<0.01$ for pairwise comparisons versus WT $+\mathrm{H}_{2} \mathrm{O}_{2}$ (Tukey's post hoc test). (b) Representative western blots showing phosphorylated $\mathrm{Src}$ (pSrc) and total Src (Src) in the brains of the indicated genotypes in the absence/presence of the indicated inhibitors. Brain lysates were incubated $1 \mathrm{~h}$ in the absence/presence of $1.0 \mathrm{mM} \mathrm{H} \mathrm{H}_{2}$ and the absence/presence of $200 \mathrm{nM}$ Cyclo or $10 \mathrm{nM}$ PND-1186 (PND). The reactions were stopped by adding sample buffer to the lysates. Quantifications of three experiments with non- $\mathrm{Tg}$ and $\mathrm{Tg}-\mathrm{C} 73 \mathrm{~A}$ are shown in the lower panel and are normalized to non- $\mathrm{Tg}+\mathrm{H}_{2} \mathrm{O}_{2} \cdot P=5.3 \times 10^{-8}$ (one-way ANOVA). ${ }^{* *} P<0.01$ for pairwise comparisons versus non- $\mathrm{Tg}$ $+\mathrm{H}_{2} \mathrm{O}_{2}$ (Tukey's post hoc test). Quantifications of two experiments with Tg-WT are shown in the lower panel and are normalized to Tg-WT+ $\mathrm{H}_{2} \mathrm{O}_{2} . P=1.9 \times 10^{-7}$ (one-way ANOVA). ${ }^{*} P<0.01$ for pairwise comparisons versus $\mathrm{Tg}-\mathrm{WT}+\mathrm{H}_{2} \mathrm{O}_{2}$ (Tukey's post hoc test)

a
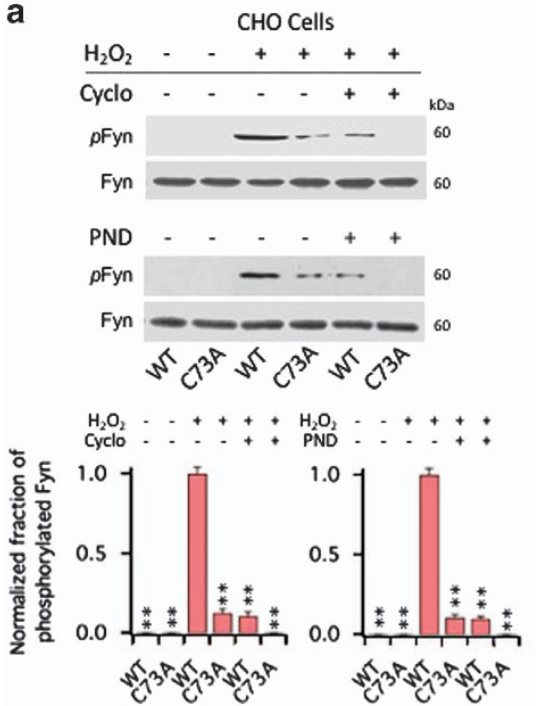

b
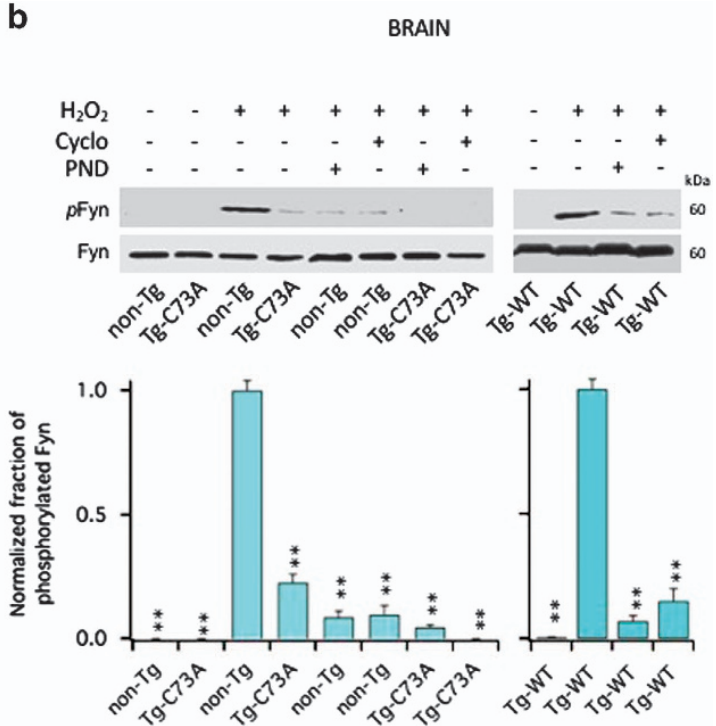

Figure 6 Cyclo and PND-1186 inhibit Fyn activation induced by oxidation of KCNB1. (a) Representative western blots showing phosphorylated Fyn at tyr530 (pFyn) and total Fyn (Fyn) in $\mathrm{CHO}$ cells transfected with WT or C73A in the absence/presence of the indicated inhibitors. Fyn protein was detected into a single, $\sim 60 \mathrm{kDa}$ band. Cells were oxidized with $1.0 \mathrm{mM} \mathrm{H}_{2} \mathrm{O}_{2}$ for 5 min and then incubated in control media or in media containing $200 \mathrm{nM}$ Cyclo or $10 \mathrm{nM} \mathrm{PND}-1186$ (PND) for $1 \mathrm{~h}$ before lysis. Quantifications of three experiments are shown in the lower panel and are normalized to $\mathrm{WT}+\mathrm{H}_{2} \mathrm{O}_{2} \cdot P=1.3 \times 10^{-6}$ and $7.7 \times 10^{-7}$ for Cyclo and PND-1186, respectively (one-way analysis of variance (ANOVA)). ${ }^{* *} \mathrm{P}<0.01$ for pairwise comparisons versus WT+ $\mathrm{H}_{2} \mathrm{O}_{2}$ (Tukey's post hoc test). (b) Representative western blots showing phosphorylated Fyn (pSrc) and total Fyn (Fyn) in the brains of the indicated genotypes in the absence/presence of Cyclo or PND-1186. Brain lysates were incubated $1 \mathrm{~h}$ in the absence/presence of $1.0 \mathrm{mM} \mathrm{H}_{2} \mathrm{O}_{2}$ and the absence/presence of $200 \mathrm{nM}$ Cyclo or $10 \mathrm{nM}$ PND-1186 (PND). The reactions were stopped by adding sample buffer to the lysates. Quantifications of three experiments with non- $\mathrm{Tg}$ and $\mathrm{Tg}-\mathrm{C} 73 \mathrm{~A}$ are shown in the lower panel and are normalized to non- $\mathrm{Tg}+\mathrm{H}_{2} \mathrm{O}_{2} . P=9.8 \times 10^{-8}$ (one-way ANOVA). ${ }^{* *} P<0.01$ for pairwise comparisons versus non-Tg $+\mathrm{H}_{2} \mathrm{O}_{2}$ (Tukey's post hoc test). Quantifications of two experiments with Tg-WT are shown in the lower panel and are normalized to Tg-WT+ $\mathrm{H}_{2} \mathrm{O}_{2} . P=1.5 \times 10^{-4}$ (one-way ANOVA). ${ }^{* *} P<0.01$ for pairwise comparisons versus Tg-WT+ $\mathrm{H}_{2} \mathrm{O}_{2}$ (Tukey's post hoc test) 
therefore the involvement of other Src family members in the apoptotic pathway activated by oxidation of KCNB1 cannot be ruled out.

Activation of the integrin-FAK-Src cascade was modest in the presence of C73A channels even though they normally interacted with integrin- $a_{5}$. The only known difference between WT and C73A channels is that oligomerization is negligible in the latter. This leads us to conclude that the transition between the non-oxidized and oxidized state of KCNB1 activates integrin signaling. KCNB1 oligomers are poorly internalized and tend to build up in the membrane. ${ }^{7}$ Thus oligomerization might favor integrin clustering, thereby facilitating the recruitment and activation of FAK and Src kinases. ${ }^{22,23}$ In the $\mathrm{Tg}-\mathrm{C} 73 \mathrm{~A}$ brains, the exogenous C73A-HA subunits form heteromeric complexes with endogenous KCNB1 subunits. The C73A mutation is dominant negative so that KCNB1 oligomerization in markedly reduced in the Tg-C73A brains. ${ }^{9}$ The remaining activity of integrin signaling in the presence of C73A may therefore be due to residual oligomerization, or alternatively, KCNB1-independent mechanisms. Future studies will address this important point.

KCNB1-induced phosphorylation of Src kinases leads to the recruitment/activation of JNK kinases, which cause oxidative stress and apoptosis by presumably targeting mitochondria. ${ }^{7}$ However, also KCNB1 channels provide a substrate for Src kinases. ${ }^{29}$ The zinc and calcium ions that are released into the cell's cytoplasm in conditions of oxidative stress trigger an apoptotic insertion of KCNB1 channels into the plasma membrane via phosphorylation of tyr124 by Src kinases and of ser800 by p38 mitogen-activated protein kinases. ${ }^{29,30}$ Modulation of KCNB1 by p38 kinases has been shown to have a role in conditions, such as hepatitis $C$ and cancer. ${ }^{31-33}$ Evidence indicates that phosphorylation of tyr124 facilitates phosphorylation of ser800 and further that phosphorylation of the latter is decreased in C73A channels without affecting tyr124. ${ }^{30}$ Even though the oligomerization and insertion mechanisms are thought to be uncoupled and to be active during distinct phases of the apoptotic process, ${ }^{18}$ it is possible that integrins may indirectly affect the phosphorylation of KCNB1 by p38 in pathological conditions through their modulation of Src signaling.

A large body of evidence indicates that many types of $\mathrm{K}^{+}$ channels, including $\mathrm{Ca}^{2+}$-activated $\mathrm{K}^{+}(\mathrm{KCa})$ channels, ${ }^{34}$ G-protein-coupled inwardly rectifying $\mathrm{K}^{+}$(GIRK) channels, ${ }^{35}$ human ether-a-go-go-related gene (HERG) $\mathrm{K}^{+}$channels $^{36}$ and voltage-gated $1.3 \mathrm{~K}^{+}$(Kv1.3) channels, ${ }^{37}$ form macromolecular complexes with integrins. These interactions have important roles for channel localization and regulation. Also KCNB1-FAK signaling - presumably through integrins - is involved in cell adhesion and migration in pathological conditions such as wound repair ${ }^{19}$ and migration of bone marrow mesenchymal stem cells to the site of the injury. ${ }^{20}$ Although the studies presented here add additional support to the notion that integrins are general partners of $\mathrm{K}^{+}$channels, they also reveal an unexpected 'antagonistic pleiotropic' nature ${ }^{38}$ of those interactions that appear to be beneficial under certain pathophysiological conditions and deleterious under other conditions, such as TBI and Alzheimer's disease. ${ }^{9,17}$ Integrins have many important signaling functions that range from the regulation of cell growth to apoptosis ${ }^{22}$ and the family of Src tyrosine kinases is a hub for many signal transduction pathways. The mechanisms that determine a specific outcome of the integrin/FAK/Src signaling are poorly understood and await further investigation.

\section{Conclusions}

The major conclusions of this study are:

- KCNB1 channels form macromolecular complexes with integrins. These complexes are detected in neurons of the brain and in $\mathrm{CHO}$ cells heterologously expressing KCNB1. Thus the ability to assemble with integrins appears to be an intrinsic property of KCNB1 channels.

- FAK kinases transduce the apoptotic signal of integrins, which lack catalytic activity. It seems plausible that KCNB1 oligomerization promotes integrin clustering, an obligated step toward recruitment of FAK.

- The activation of FAK leads to the recruitment/activation of Src tyrosine kinases and, in particular, of Fyn. However, it is possible that other members of the Src family may contribute to the same signaling pathway.

- These studies underscore a non-conducting function of a $\mathrm{K}^{+}$channel that may have implications for a series of conditions ranging from normal aging to neurodegenerative disease.

\section{Material and Methods}

Reagents. Anti-Src (clone 2108), anti-pSrc at tyr416 (clone 2101), anti-FAK (clone 3285) and anti-pFAK at tyr397 (clone 3283) were purchased from Cell Signaling Technology (Danvers, MA, USA). Anti-Fyn (clone MA1-19331) and antipFyn at tyr530 (clone PA5-38115) were purchased from Thermo Fisher (Waltham, MA, USA). Anti-HA (clone H6908) was purchased form Sigma-Aldrich (St. Louis, MO, USA). Anti-Kv2.1 (clone K89/34) was purchased from NeuroMab (University of California Davis), UC Davis (Davis, CA, USA)/NIH (Bethesda, MA, USA). Anti-Integrin- $\alpha 5$ (clone sc-10729) and goat anti-rabbit lgG-R (clone sc-2091) were purchased from Santa Cruz biotechnology (Dallas, TX, USA). Cyclo (-RGDfK) dissolved in water (100 mM) and PND-1186 dissolved in dimethyl sulfoxide (DMSO) (100 mM) were purchased from APExBIO (Houston, TX, USA).

Biochemistry and immunohistochemistry. The detailed procedures are previously described. ${ }^{9,39}$

In vitro coimmunoprecipitations: $\mathrm{CHO}$ cells (CHO-K1) were grown in DMEM medium and transfected in 6-cm dishes with Lipofectamine 2000 (Invitrogen, Carlsbad, CA, USA). For each transfection, $20 \mu \mathrm{l}$ of transfection reagent and a total of $4 \mu \mathrm{g}$ of plasmid DNA were used. Twenty-four hours following transfection, cells were lysed with $1 \mathrm{ml}$ of RIPA buffer (10 mM Tris-Cl pH 8.0, $140 \mathrm{mM} \mathrm{NaCl}, 1 \mathrm{mM}$ EDTA, $1 \%$ Triton X-100, $0.1 \%$ sodium deoxycholate, $0.1 \%$ SDS, 1 mM PMSF) and protease cocktail inhibitors (Sigma-Aldrich). Cell lysates were centrifuged for $20 \mathrm{~min}$ at $4{ }^{\circ} \mathrm{C}$ and the supernatant was mixed with $2 \mu \mathrm{g}$ of integrin- $\alpha 5$ antibody at $4{ }^{\circ} \mathrm{C}$ overnight. Then protein A/G agarose beads (Santa Cruz Biotechnology; $30 \mu \mathrm{l}$ of $50 \%$ bead slurry) were added and incubated for $2 \mathrm{~h}$ at $4{ }^{\circ} \mathrm{C}$. Samples were washed with RIPA buffer five times and the bound proteins were recovered incubating in SDS sample buffer at $100^{\circ} \mathrm{C}$ for $5 \mathrm{~min}$. Proteins were resolved by $8 \%$ SDS-PAGE and transferred onto a PVDF membrane. After blocking in a 5\% solution of non-fat milk in $0.1 \%$ Tween 20 -phosphate-buffered saline (PBS) (PBST), membranes were probed with HA antibody or Kv2.1 antibody conjugated to HRP, washed with PBST and incubated with POD chemiluminescence substrates (Roche, Branchburg, NJ, USA) and exposed.

In vivo coimmunoprecipitations: All experimental protocols involving animals were approved by the Rutgers University IACUC Committee. Frozen, half sagittal brains were homogenized with a glass tissue grinder in lysis buffer $(0.32 \mathrm{M}$ sucrose, 
$5 \mathrm{mM}$ Tris-Cl pH 6.8, 0.5 mM EDTA, $1 \mathrm{mM}$ PMSF) and protease inhibitor cocktail. Samples were centrifuged at 2000 r.p.m. for $10 \mathrm{~min}$ and the supernatant was used for biochemical analysis. Protein content was quantified with the Bradford colorimetric assay (Sigma). One milligram of brain lysates were incubated at $4{ }^{\circ} \mathrm{C}$ overnight with $2 \mu \mathrm{g}$ of integrin- $\alpha 5$ antibody. Then protein $\mathrm{A} / \mathrm{G}$ agarose beads ( $30 \mu \mathrm{l}$ of $50 \%$ bead slurry) were added and incubated for $2 \mathrm{~h}$ at $4{ }^{\circ} \mathrm{C}$. Samples were centrifuged for $30 \mathrm{~s}$ at $4{ }^{\circ} \mathrm{C}$, and the pellet was washed five times in cell lysis buffer. The pellet was resuspended with $50 \mu \mathrm{l} 2 \times$ SDS Laemmli buffer, heated at $100^{\circ} \mathrm{C}$ for $10 \mathrm{~min}$ and centrifuged for $1 \mathrm{~min}$ at $14000 \times \mathrm{g}$. The sample was loaded on $8 \%$ SDS-PAGE gel and immunoblotted with either HA or Kv2.1 antibody.

Colocalization: For colocalization experiments, the human KCNB1 cDNA was subcloned into the pEGFP-N1 vector (Clontech-Takara, Mountain View, CA, USA). Thus $\mathrm{CHO}$ cells were transiently transfected with pEGFP-N1-WT or pEGFP-N1C73A by Lipofectamine 2000 as described. Twenty-four hours later, cells were washed once with PBS and fixed with paraformaldehyde (4\% in PBS) for $15 \mathrm{~min}$ at room temperature. After being fixed, cells were washed three times for 5 min with PBS and blocked for $1 \mathrm{~h}$ at room temperature with $5 \%$ normal goat serum in PBS plus $0.3 \%$ Triton $X-100$. Cells were incubated with integrin- $\alpha_{5}$ antibody overnight at $4^{\circ} \mathrm{C}$. After washing three times for 5 min with PBS, cells were incubated with goat anti-rabbit lgG-R (Santa Cruz Biotechnology, sc-2091) for $1 \mathrm{~h}$ at room temperature. After being washed three times for $5 \mathrm{~min}$ with PBS, slides were mounted in Vectashield Antifade Mounting Medium with DAPI mounting buffer (Vector Laboratories, Burlingame, CA, USA) and stored at $4{ }^{\circ} \mathrm{C}$. Staining was visualized with a Zeiss Axiophot microscope (Thornwood, NY, USA).

Biochemical experiments were analyzed using the ImageJ software (NIH).

Preparation of hippocampal neuronal cultures. The detailed procedure was previously described. ${ }^{9}$ Briefly, hippocampi were obtained from time-mated embryonic day 16 (E16) mice and killed by $\mathrm{CO}_{2}$ asphyxiation. Hippocampal tissue from individual embryos was mechanically triturated in Neurobasal medium containing B27 (Invitrogen, Grand Island, NY, USA) and glutamine and plated in two $35 \mathrm{~mm}$ poly-D-lysine-coated petri dishes at $\sim 350000$ cells/dish $(1.5 \mathrm{ml}$ medium dish). Cultures were maintained in Neurobasal medium at $37^{\circ} \mathrm{C}$ in a $95 \%$ air $/ 5 \%$ $\mathrm{CO}_{2}$ humidified incubator and contained virtually pure neurons. Tail samples from individual embryos were processed for genotyping.

Apoptosis assays. The cells ( $\mathrm{CHO}$ cells transiently transfected as described above or primary hippocampal neurons) were washed with PBS and exposed to $1 \mathrm{mM} \mathrm{H}_{2} \mathrm{O}_{2}$ for $5 \mathrm{~min}$. The cells were washed two times with PBS and incubated for $6 \mathrm{~h}$ in fresh medium. Then the cells were washed with PBS and incubated for 15 min with Annexin-V labeling buffer solution $(20 \mu$ l of Annexin-V-Fluos and $20 \mu l$ of propidium iodide in $1 \mathrm{ml}$ of incubation buffer provided by the kit. Propidium iodide detects both necrotic and apoptotic cells. The number of cells dying by necrosis varied between 3 and $7 \%$ in average) according to kit's instructions (Annexin-VFLUOS staining kit no. 11858777001, Sigma). Cells were mounted on a Leica DMIRB inverted microscope (Leica, Buffalo Grove, IL, USA) equipped with a digital camera and photographed (6-8 images/culture) for subsequent analysis.

Experiments were performed blind. The fluorescence of Annexin- $V$ in each image was calculated using the ImageJ software $(\mathrm{NIH})$.

Dose-response curve fitting. Dose-response curves were fitted to a sigmoidal function:

$$
Y=A_{0}+\frac{A_{1}}{1+\operatorname{EXP}\left(\frac{I C_{50}-X}{A_{2}}\right)}
$$

where $Y$ is the fraction of apoptotic cells normalized to give 1 in the presence of $\mathrm{H}_{2} \mathrm{O}_{2}$ alone, $A_{0}, A_{1}$ and $A_{2}$ are constants and $X$ is the concentration of inhibitor expressed in $\mathrm{nM}$.

Statistical analysis. Quantitative data are presented as mean \pm S.E.M. All experiments were repeated in triplicate if not otherwise stated. The level of significance, assumed at the $95 \%$ confidence limit or greater $(P<0.05)$, was calculated using one-way ANOVA with a Tukey's post hoc test (http://astatsa.com/ OneWay_Anova_with_TukeyHSD).

\section{Conflict of Interest}

The authors declare no conflict of interest.
Acknowledgements. We thank Dr Shuang Liu for critical reading of the manuscript. This work was supported by an NSF grant (1456675) and an NIH grant (1R21NS096619-01) to FS.

1. Murakoshi $\mathrm{H}$, Trimmer JS. Identification of the Kv2.1 K+ channel as a major component of the delayed rectifier K+ current in rat hippocampal neurons. J Neurosci 1999; 19: 1728-1735.

2. Trimmer JS. Expression of Kv2.1 delayed rectifier $\mathrm{K}+$ channel isoforms in the developing rat brain. FEBS Lett 1993; 324: 205-210.

3. Thiffault I, Speca DJ, Austin DC, Cobb MM, Eum KS, Safina NP et al. A novel epileptic encephalopathy mutation in KCNB1 disrupts Kv2.1 ion selectivity, expression, and localization. J Gen Physiol 2015; 146: 399-410.

4. Torkamani A, Bersell K, Jorge BS, Bjork RL Jr, Friedman JR, Bloss CS et al. De novo KCNB1 mutations in epileptic encephalopathy. Ann Neurol 2014; 76: 529-540.

5. Saitsu H, Akita T, Tohyama J, Goldberg-Stern H, Kobayashi Y, Cohen R et al. De novo KCNB1 mutations in infantile epilepsy inhibit repetitive neuronal firing. Sci Rep 2015; 5: 15199.

6. Speca DJ, Ogata G, Mandikian D, Bishop HI, Wiler SW, Eum K et al. Deletion of the Kv2.1 delayed rectifier potassium channel leads to neuronal and behavioral hyperexcitability. Genes Brain Behav 2014; 13: 394-408.

7. Wu X, Hernandez-Enriquez B, Banas M, Xu R, Sesti F. Molecular mechanisms underlying the apoptotic effect of KCNB1 K+ channel oxidation. J Biol Chem 2013; 288: 4128-4134.

8. Cotella D, Hernandez-Enriquez B, Wu X, Li R, Pan Z, Leveille J et al. Toxic role of K+ channel oxidation in mammalian brain. $J$ Neurosci 2012; 32: 4133-4144.

9. Yu W, Parakramaweera R, Teng S, Gowda M, Sharad Y, Thakker-Varia S et al. Oxidation of KCNB1 potassium channels causes neurotoxicity and cognitive impairment in a mouse model of traumatic brain injury. J Neurosci 2016; 36: 11084-11096.

10. Pal S, Hartnett KA, Nerbonne JM, Levitan ES, Aizenman E. Mediation of neuronal apoptosis by Kv2.1-encoded potassium channels. J Neurosci 2003; 23: 4798-4802.

11. Oddo S, Caccamo A, Shepherd JD, Murphy MP, Golde TE, Kayed R et al. Triple-transgenic model of Alzheimer's disease with plaques and tangles: intracellular Abeta and synaptic dysfunction. Neuron 2003; 39: 409-421.

12. Smith IF, Hitt B, Green KN, Oddo S, LaFerla FM. Enhanced caffeine-induced Ca2+ release in the 3xTg-AD mouse model of Alzheimer's disease. J Neurochem 2005; 94: 1711-1718.

13. Sensi SL, Rapposelli IG, Frazzini V, Mascetra N. Altered oxidant-mediated intraneuronal zinc mobilization in a triple transgenic mouse model of Alzheimer's disease. Exp Gerontol 2008; 43: 488-492.

14. Yao J, Irwin RW, Zhao L, Nilsen J, Hamilton RT, Brinton RD. Mitochondrial bioenergetic deficit precedes Alzheimer's pathology in female mouse model of Alzheimer's disease. Proc Natl Acad Sci USA 2009; 106: 14670-14675.

15. Chou JL, Shenoy DV, Thomas N, Choudhary PK, Laferla FM, Goodman SR et al. Early dysregulation of the mitochondrial proteome in a mouse model of Alzheimer's disease. J Proteomics 2011; 74: 466-479.

16. McManus MJ, Murphy MP, Franklin JL. The mitochondria-targeted antioxidant MitoQ prevents loss of spatial memory retention and early neuropathology in a transgenic mouse model of Alzheimer's disease. J Neurosci 2011; 31: 15703-15715.

17. Frazzini V, Guarnieri S, Bomba M, Navarra R, Morabito C, Mariggio MA et al. Altered Kv2.1 functioning promotes increased excitability in hippocampal neurons of an Alzheimer's disease mouse model. Cell Death Dis 2016; 7: e2100.

18. Sesti $\mathrm{F}$, Wu X, Liu S. Oxidation of $\mathrm{KCNB} 1 \mathrm{~K}(+)$ channels in central nervous system and beyond. World J Biol Chem 2014; 5: 85-92.

19. Wei JF, Wei L, Zhou X, Lu ZY, Francis K, Hu XY et al. Formation of Kv2.1-FAK complex as a mechanism of FAK activation, cell polarization and enhanced motility. J Cell Physiol 2008; 217: 544-557.

20. Hu X, Wei L, Taylor TM, Wei J, Zhou X, Wang JA et al. Hypoxic preconditioning enhances bone marrow mesenchymal stem cell migration via Kv2.1 channel and FAK activation. Am J Physiol Cell Physiol 2011; 301: C362-C372.

21. Sieg DJ, Hauck CR, Schlaepfer DD. Required role of focal adhesion kinase (FAK) for integrin-stimulated cell migration. J Cell Sci 1999; 112(Pt 16): 2677-2691.

22. Desgrosellier JS, Cheresh DA. Integrins in cancer: biological implications and therapeutic opportunities. Nat Rev Cancer. 2010; 10: 9-22.

23. Mitra SK, Hanson DA, Schlaepfer DD. Focal adhesion kinase: in command and control of cell motility. Nat Rev Mol Cell Biol 2005; 6: 56-68.

24. Konig H, Copland M, Chu S, Jove R, Holyoake TL, Bhatia R. Effects of dasatinib on SRC kinase activity and downstream intracellular signaling in primitive chronic myelogenous leukemia hematopoietic cells. Cancer Res 2008; 68: 9624-9633.

25. Liu DZ, Sharp FR, Van KC, Ander BP, Ghiasvand R, Zhan X et al. Inhibition of SRC family kinases protects hippocampal neurons and improves cognitive function after traumatic brain injury. J Neurotrauma 2014; 31: 1268-1276.

26. Shirazi SK, Wood JG. The protein tyrosine kinase, fyn, in Alzheimer's disease pathology. Neuroreport 1993; 4: 435-437.

27. Schmid RS, Anton ES. Role of integrins in the development of the cerebral cortex. Cereb Cortex 2003; 13: 219-224.

28. Mitra SK, Schlaepfer DD. Integrin-regulated FAK-Src signaling in normal and cancer cells. Curr Opin Cell Biol 2006; 18: 516-523.

29. McCord MC, Aizenman E. Convergent $\mathrm{Ca} 2+$ and $\mathrm{Zn} 2+$ signaling regulates apoptotic Kv2.1 K+ currents. Proc Natl Acad Sci USA 2013; 110: 13988-13993.

30. He K, McCord MC, Hartnett KA, Aizenman E. Regulation of pro-apoptotic phosphorylation of Kv2.1 K+ channels. PLOS ONE 2015; 10: e0129498. 
31. Mankouri J, Dallas ML, Hughes ME, Griffin SD, Macdonald A, Peers C et al. Suppression of a pro-apoptotic $\mathrm{K}+$ channel as a mechanism for hepatitis $\mathrm{C}$ virus persistence. Proc Natl Acad Sci USA 2009; 106: 15903-15908.

32. Amako Y, Igloi Z, Mankouri J, Kazlauskas A, Saksela K, Dallas M et al. Hepatitis C virus NS5A inhibits mixed lineage kinase 3 to block apoptosis. J Biol Chem 2013; 288: 24753-24763.

33. Al-Owais MM, Scragg JL, Dallas ML, Boycott HE, Warburton P, Chakrabarty A et al. Carbon monoxide mediates the anti-apoptotic effects of heme oxygenase- 1 in medulloblastoma DAOY cells via K+ channel inhibition. J Biol Chem 2012; 287: 24754-24764.

34. Girault A, Chebli J, Prive A, Trinh NT, Maille E, Grygorczyk R et al. Complementary roles of KCa3.1 channels and beta1-integrin during alveolar epithelial repair. Respir Res 2015; 16: 100.

35. McPhee JC, Dang YL, Davidson N, Lester HA. Evidence for a functional interaction between integrins and G protein-activated inward rectifier K+ channels. J Biol Chem 1998; 273: 34696-34702.

36. Cherubini A, Hofmann G, Pillozzi S, Guasti L, Crociani O, Cilia E et al. Human ether-a-go-gorelated gene 1 channels are physically linked to beta1 integrins and modulate adhesiondependent signaling. Mol Biol Cell 2005; 16: 2972-2983.

37. Levite M, Cahalon L, Peretz A, Hershkoviz R, Sobko A, Ariel A et al. Extracellular K(+) and opening of voltage-gated potassium channels activate $T$ cell integrin function: physical and functional association between Kv1.3 channels and beta1 integrins. J Exp Med 2000; 191: $1167-1176$.
38. Williams GC. Pleiotropy, natural selection and the evolution of senescence. Evolution 1957; 11: $398-411$.

39. Cotella D, Hernandez-Enriquez B, Duan Z, Wu X, Gazula VR, Brown MR et al. An evolutionarily conserved mode of modulation of Shaw-like K(+) channels. FASEB J 2013; 27: 1381-1393.

(c) (i) Cell Death and Disease is an open-access journal published by Nature Publishing Group. This work is licensed under a Creative Commons Attribution 4.0 International License. The images or other third party material in this article are included in the article's Creative Commons license, unless indicated otherwise in the credit line; if the material is not included under the Creative Commons license, users will need to obtain permission from the license holder to reproduce the material. To view a copy of this license, visit http://creativecommons.org/licenses/by/4.0/

(C) The Author(s) 2017 